\title{
Training doctors in perioperative medicine for older people undergoing surgery (POPS): an innovative foundation placement 篦
}

\author{
Authors: Sarah Barber, ${ }^{A}$ Edward Singleton, ${ }^{B}$ Judith SL Partridge ${ }^{C}$ and Jugdeep K Dhesi ${ }^{D}$
}

KEYWORDS: Perioperative medicine for older people undergoing surgery (POPS), foundation doctor programme, medical education and training, perioperative medicine

\section{Introduction}

In 2013, the Future Hospital Commission published a report outlining radical steps required to meet the needs of a changing patient population. This highlighted the need for medical care for all hospital inpatients, not just those on medical wards, stipulating that medical education and training must equip doctors 'with the knowledge and skills to manage the future demographic of patients. ${ }^{1}$ The Shape of Training Review, published in the same year, further emphasised the need for doctors to provide general care in broad specialties across a range of settings; whole patient, whole pathway medicine. ${ }^{2}$ To achieve this service provision, the review asserted that training should reflect the needs of the changing patient population. This requires training and services to be tailored to an older population where multimorbidity and frailty are frequently observed. .,4 $^{3}$

This is particularly true in the case of surgical patients, where the population is ageing faster than the general population (1.8 vs 0.7 years per annum). It is estimated that by 2030 , one-fifth

\footnotetext{
Authors: Afoundation doctor, perioperative medicine for older people undergoing surgery (POPS) team, Guy's and St Thomas' NHS Foundation Trust, London, UK; ${ }^{\text {B }}$ foundation doctor, perioperative medicine for older people undergoing surgery (POPS) team, Guy's and St Thomas' NHS Foundation Trust, London, UK; ' Consultant geriatrician, perioperative medicine for older people undergoing surgery (POPS) team, Guy's and St Thomas' NHS Foundation Trust, London, UK and honorary senior lecturer, Faculty of Life Sciences and Medicine, King's College London, London, UK; ${ }^{\mathrm{D}}$ consultant geriatrician, perioperative medicine for older people undergoing surgery (POPS) team, Guy's and St Thomas' NHS Foundation Trust, London, UK and honorary reader, Faculty of Life Sciences and Medicine, King's College London, London, UK and honorary associate professor, Division of Surgery and Interventional Science, University College London, London, UK
}

of people aged 75 years and older will undergo surgery every year. ${ }^{5}$ While older patients have much to benefit from surgery in terms of symptom control and improved longevity, they remain at higher risk of adverse postoperative outcomes including postoperative complications, longer length of stay and functional deterioration resulting in institutionalisation. ${ }^{6,7}$

The emerging specialty of perioperative medicine aims to provide quality care for this high risk population through evidencebased medicine delivered throughout the surgical pathway. The Centre for Perioperative Care, launched in 2019, is a crossspecialty, multidisciplinary partnership led by the Royal College of Anaesthetists, which aims to orientate surgical services to the changing surgical population. One model of care advocated by this initiative involves the use of comprehensive geriatric assessment and optimisation (CGA) methodology employed through multidisciplinary, interspecialty perioperative medicine for older people undergoing surgery (POPS) services. ${ }^{8,9}$ In addition to clinical care, POPS services offer the opportunity to train clinicians in perioperative medicine tailored to a high-risk older population. Such an initiative is aligned with the perioperative agenda, recommendations from the Future Hospital Commission and the Shape of Training Review.

This article describes an innovative approach to training for foundation doctors in perioperative medicine for older people.

\section{The POPS foundation placement}

POPS is a CGA-based, geriatrician-led service at Guy's and St Thomas' Hospital, established in 2003. Foundation trainees (doctors in the first and second years of clinical practice after completing their medical degree) have been part of the multidisciplinary team since 2015. The POPS foundation placement follows a modular structure (Table 1) that aims to deliver generic capabilities and cross-specialty competencies (Table 2) based on a curriculum (see supplementary material S1) co-designed with foundation doctors and iteratively modified through weekly-feedback fora. ${ }^{10}$ The modular structure allows the foundation doctor to focus on inpatient medicine ( 2 months) and community facing medicine with sessions in outpatient clinic, theatre and community rehabilitee units ( 2 months). On-call commitment and annual leave is distributed throughout.

\section{Evaluation of the POPS foundation programme}

Following its establishment in 2015, the POPS Foundation programme underwent a mixed-methods evaluation. ${ }^{10}$ Outcome 


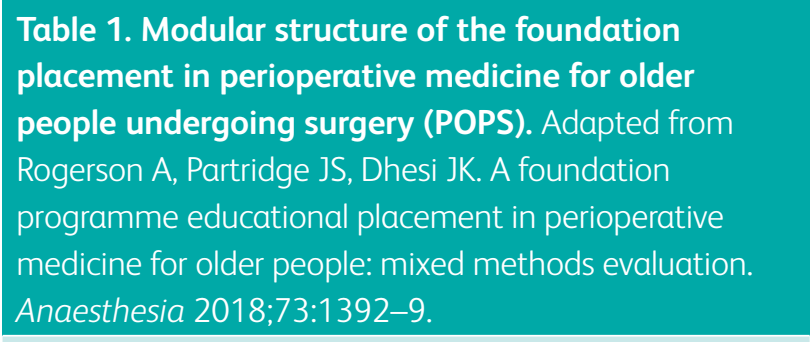

Inpatient Medical ward rounds.

Joint medical and surgical ward rounds.

Board rounds/MDMs.

Medical case management.

Family meetings.

Outpatient POPS preoperative CGA and optimisation clinic.
and

community

Surgical clinics (matched to surgical subspecialty).

Scheduled theatre sessions with experience in anaesthesia and surgery.

Ward rounds at community amputee rehabilitation centre.

On call 2-week block of surgical and medical on-call commitment.

Formal Generic hospital-delivered core curriculum education foundation training.

Weekly trust grand round.

Weekly geriatric medicine departmental teaching.

Weekly consultant or registrar-delivered POPS teaching session (1 hour) based on POPS curriculum.

Opportunities to attend other relevant teaching sessions and conferences.

Formal teaching on quality improvement methodology, presenting and publishing findings.

CGA = comprehensive geriatric assessment; $\mathrm{MDMs}$ = multidisciplinary meetings; $\mathrm{POPS}=$ perioperative medicine for older people undergoing surgery.

measures included core foundation competencies, learning objectives in perioperative medicine and quality of training. Over a 2-year period, all POPS foundation trainees $(n=68)$ achieved full portfolio sign off (indicating adequate opportunity to practice and record core foundation competencies). In addition, job satisfaction was also deemed to be higher in POPS foundation doctors compared with foundation trainees in standard surgical posts. The authors concluded that the placement 'provides effective and enjoyable training in line with national recommendations regarding "whole patient medicine."' The learning experiences of two current POPS foundation trainees are illustrated below.

Edward (foundation year 1):

POPS has grown my confidence in decision making and ownership of patient care, which are essential foundations for any medical career. Through regular participation in multidisciplinary meetings, my clinical work is continually evaluated and improved. An invaluable learning opportunity.
Table 2. Perioperative medicine for older people

undergoing surgery rotation competencies and

generic capabilities / transferable skills

\section{Competencies}

Core

Evidence-based medicine.

Long-term condition management.

Recognition and resuscitation of the acutely unwell medical patient in the context of medical and surgical complexity/uncertainty.

Geriatric Comprehensive geriatric assessment.

medicine Multimorbidity and frailty.

Cognitive disorders.

Advanced care planning.

Perioperative Preoperative risk assessment and medicine optimisation.

Perioperative planning.

Shared decision making.

Postoperative medical management, rehabilitation and discharge planning.

Surgery/ Physiological assessment to inform anaesthesia anaesthetic management.

Surgical stress response.

Pathway of care.

\section{Generic capabilities / transferable skills}

Communication Collaborative interspecialty and and multidisciplinary working.

professionalism Patient and relative communication:

> advanced care planning conversations / shared decision making

$>$ promotion of self-management and lifestyle changes.

Multidisciplinary team meetings.

Teamwork Within POPS team (consultant, registrar, nurse specialists, allied healthcare professionals).

Joint working between POPS, surgery, anaesthesia, critical care, palliative medicine, oncology.

Leadership Patient advocacy.

Chairing MDMs.

Quality Compulsory completion of QI project with improvement dedicated sessions to complete this.

Formal teaching on QI methodology.

Formal teaching on presenting and publishing findings.

MDMs = multi-disciplinary meetings; POPS = perioperative care for older people undergoing surgery; QI = quality improvement. 
I now see that discussing risk is more than a tick box exercise; it is an opportunity to face realities and for the patient to be able to make truly informed decisions. In addition, I have realised that a key part of the optimisation and recovery process involves empowering and supporting patients to take ownership of their own health.

\section{Conclusions}

POPS is an example of a perioperative training programme. The POPS foundation placement not only delivers training on core foundation competencies and specialty-specific learning points, but also promotes key transferable skills that are essential to a cadre of doctors required to deliver 'whole patient medicine'. POPS instils in its trainees the importance of comprehensive, evidencebased, patient-centred care and clear communication with patients, families and healthcare professionals to inform shared decision making. Juniors are supported to become competent in managing complex, multimorbid patients in a variety of settings across the whole patient pathway. They are also encouraged to appreciate the wider context of their work in relation to demographic trends, national reports and policy drivers. Through their quality improvement work, they have the opportunity to make positive change at the local and sometimes national level. Importantly, it is also enjoyable! Innovative foundation programmes such as this are required to meet the challenges ahead =

\section{Supplementary material}

Additional supplementary material may be found in the online version of this article at www.rcpjournals.org:

S1 - Curriculum for foundation programme in perioperative medicine for older people

\section{References}

1 Future Hospital Commission. Future hospital: Caring for medical patients. London: Royal College of Physicians, 2013.

2 Shape of Training Review. Shape of Training. Securing the future of excellent patient care. London: Shape of Training, 2013. www.shapeoftraining.co.uk/static/documents/content/Shape_of_ training_FINAL_Report.pdf_53977887.pdf [Accessed 11 July 2019].

3 Kingston A, Robinson L, Booth $\mathrm{H}$ et al. Projections of multi-morbidity in the older population in England to 2035: estimates from the Population Ageing and Care Simulation (PACSim) model. Age and Ageing 2018:47:374-80.

4 Campbell AJ, Buchner DM. Unstable disability and the fluctuations of frailty. Age and Ageing 1997;26:315-8.

5 Fowler AJ, Abbott TEF, Prowle J et al. Age of patients undergoing surgery. Br J Surg 2019;106:1012-1018.

6 Polanczyk CA, Marcantonio E, Goldman L et al. Impact of age on perioperative complications and length of stay in patients undergoing noncardiac surgery. Ann Internal Med 2001;134:637-43.

7 Hamel MB, Henderson WG, Khuri SF et al. Surgical outcomes for patients aged 80 and older: morbidity and mortality from major noncardiac surgery. Journal of the American Geriatrics Society 2005;53:424-9.

8 Royal College of Anaesthetists. Perioperative medicine: The pathway to better surgical care. London: RCoA, 2015. www.rcoa. ac.uk/periopmed/vision-document [Accessed 11 July 2019].

9 Partridge JSL, Harari D, Martin FC et al. Randomized clinical trial of comprehensive geriatric assessment and optimization in vascular surgery. Br ] Surg 2017;104:679-87.

10 Rogerson A, Partridge JS, Dhesi JK. A foundation programme educational placement in perioperative medicine for older people: mixed methods evaluation. Anaesthesia 2018;73:1392-9.

Address for correspondence: Dr Edward Singleton, Guy's and St Thomas' Hospital Foundation Trust (GSTT), Great Maze Pond, London SE1 9RT, UK.

Email: edward.singleton@doctors.org.uk 